\title{
Functions in Sampling Spaces
}

\author{
Cristina Blanco \\ Depto. de Matemática, Univ. de Buenos Aires \\ Cdad. Univ., Pab. I \\ 1428 Capital Federal, Argentina \\ cblanco@dm.uba.ar \\ Carlos Cabrelli \\ Depto. de Matemática, Univ. de Buenos Aires and CONICET \\ Cdad. Univ., Pab. I \\ 1428 Capital Federal, Argentina \\ cabrelli@dm.uba.ar \\ Sigrid Heineken \\ Depto. de Matemática, Univ. de Buenos Aires and CONICET \\ Cdad. Univ., Pab. I \\ 1428 Capital Federal, Argentina \\ sheinek@dm.uba.ar
}

May 16, 2007

\begin{abstract}
Sampling theory in spaces other than the space of band-limited functions has recently received considerable attention. This is in part because the band-limitedness assumption is not very realistic in many applications. In addition, band-limited functions can have very slow decay which translates in poor reconstruction. In this article we study the sampling problem in general shift invariant spaces. We characterize the functions in these spaces and provide necessary and sufficient conditions for a function in $L^{2}(\mathbb{R})$ to belong to a sampling space. Furthermore we obtain decompositions of a sampling space in sampling subspaces. These decompositions are related with determining sets. Some examples are provided.
\end{abstract}

Key words and phrases: Shift invariant spaces, sampling spaces, frames, determining sets

2000 AMS Mathematics Subject Classification - 39A10, 42C40, 41A15 


\section{Introduction}

Sampling theory originally has been developed in the space $P_{\sigma}$ of $\sigma$ band-limited functions, i.e. the space of functions $f \in L^{2}(\mathbb{R})$ such that supp $\hat{f} \subset[-\sigma, \sigma]$, where $\sigma>0$ and

$$
\hat{f}(\omega)=\int_{-\infty}^{+\infty} f(x) e^{-2 \pi i x \omega} d x
$$

The Classical Sampling theorem states that every function $f$ in this class can be recovered from its samples $f\left(\frac{k}{2 \sigma}\right)_{k \in \mathbb{Z}}$. Furthermore the following reconstruction formula holds:

$$
f(x)=\sum_{k \in \mathbb{Z}} f\left(\frac{k}{2 \sigma}\right) \frac{2 \sigma \sin (\pi(2 \sigma x-k))}{\pi(2 \sigma x-k)},
$$

where the series on the right hand side converges uniformly and in $L^{2}(\mathbb{R})$. This theorem has been generalized in many ways. For example to answer the question whether a similar result can be obtained for the case of irregular sampling (i.e. when the samples values are not on a lattice), or when we only have average sampling values, (i.e. the function has to be recovered from the values $\left\langle f, \phi_{k}\right\rangle$, where each $\phi_{k}$ is a $L^{2}$-function that is well localized around $k$ ). There is a profuse literature on the subject. We recommend the books [BF01, [BZ04] and the survey AG01 and the references there in.

The space $P_{1 / 2}$ where the Classical Sampling Theorem holds, is in particular a Shift Invariant Space (SIS) with generating function $\psi(x)=\frac{\sin \pi x}{\pi x}$, moreover $\{\psi(\cdot-k)\}_{k \in \mathbb{Z}}$ is an orthonormal basis of $P_{1 / 2}$, in particular a frame for $P_{1 / 2}$.

Let us recall that a SIS in $L^{2}(\mathbb{R})$ is a subspace of $L^{2}(\mathbb{R})$, such that it is invariant under integer translations.

Given functions $f_{1}, f_{2}, \ldots, f_{n} \in L^{2}(\mathbb{R})$, we will denote by $S\left(f_{1}, \ldots, f_{n}\right)$, the SIS generated by the integer translates of these functions, i.e. the $L^{2}(\mathbb{R})$ - closure of the span of the set $\left\{f_{i}(\cdot-k): i=1, \ldots, n, k \in \mathbb{Z}\right\}$.

When the set $\left\{f_{i}(\cdot-k): i=1, \ldots, n, k \in \mathbb{Z}\right\}$, forms a frame of $S\left(f_{1}, \ldots, f_{n}\right)$, we will write sometimes $V\left(f_{1}, \ldots, f_{n}\right)$ instead of $S\left(f_{1}, \ldots, f_{n}\right)$, to stress this fact. It is known that every space $S\left(f_{1}, \ldots, f_{n}\right)$ contains functions $g_{1}, \ldots, g_{l}$, with $l \leq n$, such that $S\left(f_{1}, \ldots, f_{n}\right)=V\left(g_{1}, \ldots, g_{l}\right)$.

There is a natural interest in trying to obtain sampling theorems in SIS's other than spaces of band-limited functions, Wal92, [SZ99], since the bandlimitedness assumption is not realistic in numerous applications in digital signal and image processing, AG01.

Our main interest in this article is to study the structure and find characterizations of functions of $L^{2}(\mathbb{R})$ that belong to a general sampling space $V(\varphi)$. A sampling space is a space $V(\varphi)$ where the generator has special properties.

Throughout this paper we will consider the following class of sampling spaces:

Definition 1. A closed subspace $V(\varphi)$ of $L^{2}(\mathbb{R})$ is called a sampling space if there exists a function $s$ such that: 
1. The translates $\{s(\cdot-k)\}_{k \in \mathbb{Z}}$ are a frame for the space $V(\varphi)$.

2. For every sequence $\left\{c_{k}\right\}_{k \in \mathbb{Z}} \in \ell^{2}(\mathbb{Z})$ the series $\sum_{k \in \mathbb{Z}} c_{k} s(\cdot-k)$ converges pointwise to a continuous function.

3. For every $f \in V(\varphi)$,

$$
f(x)=\sum_{k \in \mathbb{Z}} f(k) s(x-k),
$$

where the convergence is in $L^{2}(\mathbb{R})$ and uniform in $\mathbb{R}$.

The function $s$ is called the sampling function of $V(\varphi)$, and is the only function in $V(\varphi)$ that satisfies equation (11). (See Lemma 3).

We found that if a function $f$ belongs to a sampling space, then $S(f)$ is a sampling space itself. We characterize the sampling function of $S(f)$ as the orthogonal projection, onto $S(f)$, of the sampling function of the original space. From here, using a characterization of sampling spaces given by Sun and Zhou SZ99, we obtain necessary and sufficient conditions for a function $f$ to belong to a sampling space.

These results can be connected with the problem of Determining Sets for sampling spaces, ACHMR03. Basically a set of functions of a finitely generated SIS is a determining set if and only if it is a set of generators (in the sense mentioned above) of the SIS. Necessary and sufficient conditions for a finite subset of a general finitely generated SIS to be a determining set have been obtained in ACHMR03. We obtain the equivalent conditions in the case of sampling spaces and we show that given a determining set of a sampling space, then the space can be decomposed as a sum of smaller sampling spaces, each of them generated by one of the functions in the determining set. We characterize all possible (countable) decompositions of a sampling space $V(\varphi)$ as a direct sum of sampling spaces.

The grammian of a function $\varphi \in L^{2}(\mathbb{R})$ is the function $G_{\varphi}(\omega)=\sum_{k \in \mathbb{Z}} \mid \hat{\varphi}(\omega+$ $k)\left.\right|^{2}$. Denote by $E_{\varphi}$ the set $E_{\varphi}=\left\{w \in \mathbb{R}: G_{\varphi}(\omega)>0\right\}$. The set $E_{\varphi}$ is periodic i.e. $E_{\varphi}=E_{\varphi}+k$ for every integer $k$. If $\psi$ is any generator of $S(\varphi)$, then $E_{\psi}=E_{\varphi}$. If $V(\varphi)$ is a sampling space, every partition of $E_{\varphi}$ into countable measurable periodic sets defines a decomposition of $V(\varphi)$ as a direct sum of sampling spaces. The sampling function of each of these spaces turns out to be the orthogonal projection of the sampling function over each of the components. This type of decompositions has been obtained in BDR94, Bow00, for SIS of $L^{2}(\mathbb{R})$. We show here that it is still valid for sampling spaces, that is, the subspaces in the decomposition are also sampling spaces.

In [SZ04, Sun and Zhou obtained sufficient conditions for a function to belong to a sampling space. The conditions are not necessary as we show in 
Example 2. We found necessary and sufficient conditions for a function to belong to a sampling space, for the case of sampling spaces with generators having Fourier transforms in $L^{1}(\mathbb{R})$. As expected, the $\sigma$ band-limited functions satisfy these conditions (see Example 1).

The paper is organized as follows: In section 2 we state the main results, in section 3 we present the proofs and section 4 is devoted to some examples.

\section{Statement of Results}

Definition 2. A sequence $\left\{\psi_{k}\right\}_{k \in \mathbb{Z}}$ is a frame for a separable Hilbert space $\mathcal{H}$ if there exist positive constants $A$ and $B$ that satisfy

$$
A\|f\|^{2} \leq \sum_{k \in \mathbb{Z}}\left|\left\langle f, \psi_{k}\right\rangle\right|^{2} \leq B\|f\|^{2} \quad \forall f \in \mathcal{H}
$$

If $\left\{\psi_{k}\right\}_{k \in \mathbb{Z}}$ satisfies the right inequality in the above formula, it is called a Bessel sequence.

For a shift invariant space $S(\psi)$ it is known (BDR94) that the integer translates of the function $\varphi$ defined by

$$
\hat{\varphi}(\omega)= \begin{cases}\frac{\hat{\psi}(\omega)}{G_{\psi}(\omega)^{\frac{1}{2}}} & \text { for } \omega \in E_{\psi} \\ 0 & \text { otherwise }\end{cases}
$$

form a tight frame of $S(\psi)$, in particular $S(\psi)=V(\varphi)$. Now we state our first theorem:

Theorem 1. Let $V(\varphi)$ be a sampling space with sampling function $s$ and $f \in$ $L^{2}(\mathbb{R})$. If $f \in V(\varphi)$ then $S(f)$ is a sampling space with sampling function $s_{f}=$ $P_{S(f)}(s)$, where $P_{S(f)}(s)$ is the orthogonal projection of $s$ onto $S(f)$. In this case $\hat{s_{f}}=\hat{s} \chi_{E_{f}}$.

We will use this result, together with the characterization of a sampling space obtained by Sun and Zhou in SZ99, to obtain necessary and sufficient conditions on a function $f$ to belong to a sampling space.

We first need the following definition:

Definition 3. For $f \in L^{2}(\mathbb{R})$ the Zak transform of $f$ is the function on $\mathbb{R}^{2}$ :

$$
Z_{f}(x, \omega)=\sum_{k \in \mathbb{Z}} f(x+k) e^{-2 \pi i k \omega} .
$$

For properties of the Zak transform see [Jan88].

Theorem 2. Assume $f \in L^{2}(\mathbb{R})$. Then $f$ belongs to a sampling space if and only if the function $h$ defined by $\hat{h}=\hat{f} / G_{f}$ in $E_{f}$ and zero otherwise, satisfies: 
1. $h$ is continuous.

2. The function $\sum_{k \in \mathbb{Z}}|h(x-k)|^{2}$ is bounded on $\mathbb{R}$.

3. There exist constants $A, B>0$ such that

$$
A \chi_{E_{f}}(\omega) \leq\left|Z_{h}(0, \omega)\right| \leq B \chi_{E_{f}}(\omega) \text { a.e. } \omega \text {. }
$$

This results can now be applied to the problem of determining sets.

Given a set $\mathcal{F}=\left\{f_{1}, \ldots, f_{m}\right\} \subset L^{2}(\mathbb{R})$ where $f_{i}$ are functions that belong to an unknown shift invariant space $V(\varphi)$, it is an important matter to be able to decide whether this set $\mathcal{F}$ is sufficient to determine $V(\varphi)$. This leads to the concept of determining sets of shift invariant spaces. In ACHMR03, the problem is solved if $\{\varphi(\cdot-k)\}_{k \in \mathbb{Z}}$ is a Riesz basis of $V(\varphi)$.

We study the problem in the case that $V(\varphi)$ is a sampling space. We give necessary and sufficient conditions on $\mathcal{F}$, needed for determining the unknown sampling space $V(\varphi)$. Moreover we decompose the sampling space $V(\varphi)$ as the sum of the sampling spaces $S\left(f_{i}\right)$. In particular the sampling function $s$ of $V(\varphi)$ can be recovered from the functions $f_{i}$.

Definition 4. Let $V(\varphi)$ be a shift invariant space. The set $\mathcal{F}=\left\{f_{1}, \ldots, f_{m}\right\} \subset$ $V(\varphi)$ is a determining set for $V(\varphi)$ if for any $g \in V(\varphi)$ there exist $\hat{\alpha_{1}}, \ldots, \hat{\alpha_{m}}$ 1-periodic measurable functions such that:

$$
\hat{g}=\hat{\alpha_{1}} \hat{f_{1}}+\cdots+\hat{\alpha_{m}} \hat{f_{m}}
$$

Theorem 3. Assume $V(\varphi)$ is a sampling space. Then $\mathcal{F}=\left\{f_{1}, \ldots, f_{m}\right\} \subset V(\varphi)$ is a determining set for $V(\varphi)$ if and only if the set

$$
Z=\left(\bigcup_{i=1}^{m} E_{f_{i}}\right) \triangle E_{\varphi}
$$

has Lebesgue measure zero (where $\triangle$ denotes the symmetric difference of sets).

Moreover, if $\mathcal{F}$ is a determining set for $V(\varphi)$, then

$$
V(\varphi)=S\left(f_{1}\right)+\cdots+S\left(f_{m}\right)
$$

From Theorem 1 we can also obtain the following decomposition of a sampling space.

Proposition 1. Let $V(\varphi)$ be a sampling space and let $\left\{E_{j}\right\}_{j \in N}$ be a partition of $E_{\varphi}$ in periodic measurable sets (i.e. $E_{\varphi}=\bigcup_{j} E_{j},\left|E_{j} \cap E_{l}\right|=0$ for all $j \neq l$, and $E_{j}+k=E_{j}$ for every integer $\left.k\right)$.

Define for $j \in \mathbb{N}, \varphi_{j}$ by $\hat{\varphi}_{j}=\hat{\varphi} \chi_{E_{j}}$. Then we have: 


$$
V(\varphi)=\bigoplus_{j} V\left(\varphi_{j}\right)
$$

where $V\left(\varphi_{j}\right)$ is a sampling space for each $j$. Furthermore, if $s$ is the sampling function of $V(\varphi)$ then the Fourier transform of the sampling function $s_{j}$ of $V\left(\varphi_{j}\right)$ is $\hat{s}_{j}=\hat{s} \chi_{E_{j}}$.

We now return to the study of the characterization of functions that belong to sampling spaces. Our results in this part are related to a recent result of Sun and Zhou SZ04. Let

$$
\mathcal{U}=\left\{f \in L^{2}(\mathbb{R}): \hat{f} \in L^{1}(\mathbb{R})\right\} .
$$

In SZ04, sufficient conditions for a function $f$ in $\mathcal{U}$ to belong to a sampling space are given. We show in Example 2 that these conditions are not necessary.

We found necessary and sufficient conditions for a function in $\mathcal{U}$ to belong to a sampling space for the case that the generator of the sampling space is in $\mathcal{U}$. Note that if the generator $\varphi$ of the sampling space $V(\varphi)$ is in $\mathcal{U}$, then any other function $\psi$ such that $V(\psi)=V(\varphi)$ is also in $\mathcal{U}$.

The conditions are stated in the following theorem:

Theorem 4. Let $f \in \mathcal{U}$. Then $f$ belongs to a sampling space $V(\varphi)$ with $\varphi \in \mathcal{U}$ if and only if $f$ satisfies:

a) The sequence $\{f(k)\}_{k \in \mathbb{Z}} \in \ell^{2}(\mathbb{Z})$,

b) there exist $A, B>0$ such that

$$
A\left|Z_{f}(0, \omega)\right|^{2} \leq G_{f}(\omega) \leq B\left|Z_{f}(0, \omega)\right|^{2} \quad \text { a.e. } \omega \in \mathbb{R}
$$

c) The integral

$$
\int_{[0,1] \cap E_{f}} \frac{\sum_{k \in \mathbb{Z}}|\hat{f}(\omega+k)|}{\left|Z_{f}(0, \omega)\right|} d \omega<+\infty,
$$

d) There exists $L>0$ such that

$$
\int_{[0,1] \cap E_{f}}\left|\frac{Z_{\hat{f}}(\omega,-x)}{Z_{f}(0, \omega)}\right|^{2} d \omega<L<+\infty \quad \forall x \in \mathbb{R} .
$$

Remark 1. We have, in fact, the following stronger version of the necessary condition in Theorem 4 :

Proposition 2. If $f \in L^{2}(\mathbb{R})$ belongs to a sampling space $V(\varphi)$ with $\varphi \in \mathcal{U}$, then $f$ satisfies $a), b), c), d$ ). 
Finally we observe that it is natural to consider the sampling problem on a general lattice $a \mathbb{Z}+b$, with $a \in \mathbb{R}_{>0}, b \in \mathbb{R}$. For this, let $T: L^{2}(\mathbb{R}) \rightarrow L^{2}(\mathbb{R})$ be an unitary operator and $V=V(\varphi)$ a sampling space. Set $W=T(V)$.

Consider $g \in W$ and call $f=T^{-1} g$. Then $f=\sum_{k} f(k) t_{k} \varphi$, where $t_{k}$ is the translation operator $t_{k} h(x)=h(x-k)$. So, $g=T f=\sum_{k} f(k)\left(T \circ t_{k}\right) \varphi=$ $\sum_{k} T^{-1}(T f)(k)\left(T \circ t_{k}\right) \varphi$. That is

$$
g=\sum_{k}\left(T^{-1} g\right)(k)\left(T \circ t_{k}\right) \varphi, \quad \forall g \in W .
$$

Let us now define the unitary dilation operator $D_{a}$ by $D_{a} f(x)=\sqrt{a} f(a x)$ and $T$ by $T=D_{a} \circ t_{b}$. Denote $\psi=T \varphi$. Then, due to the commutation relation $D_{a} t_{k}=t_{\frac{k}{a}} D_{a}$, the following sampling formula holds:

$$
\left.g=\sum_{k} g\left(\frac{k+b}{a}\right) \psi\left(x-\frac{k}{a}\right), \quad \forall g \in V_{a, b}=\overline{\operatorname{span}}\left(\left\{\psi\left(\cdot-\frac{k}{a}\right), k \in \mathbb{Z}\right\}\right)\right) .
$$

\section{Proofs}

We will first state some known results that we will use in the proofs.

In BL98] Benedetto and Li proved the following result:

Proposition 3. The sequence $\{\psi(\cdot-k)\}_{k \in \mathbb{Z}}$ is a frame for the closure in $L^{2}(\mathbb{R})$ of the space it spans if and only if there exist positive constants $A$ and $B$ that satisfy

$$
A \leq G_{\psi}(\omega) \leq B \quad \text { a.e. } \omega \in E_{\psi}
$$

Proposition 4. (Theorem 2.14 [BDR94]) Let $S(\psi)$ be a shift invariant space. $A$ function $f$ is in $S(\psi)$ if and only if $\hat{f}=r \hat{\psi}$ for some periodic function $r$ of period one, with $r \hat{\psi} \in L^{2}(\mathbb{R})$.

We will also need this known result: if the sequence $\left\{\psi_{k}\right\}_{k \in \mathbb{Z}}$ is a frame for the Hilbert space $\mathcal{H}$, then $f \in \mathcal{H}$ if and only if $f=\sum_{k \in \mathbb{Z}} c_{k} \psi_{k}$, for some $c_{k} \in \ell^{2}(\mathbb{Z})$ with convergence in $L^{2}(\mathbb{R})$.

Sun and Zhou gave the following characterization, which we will use later to determine if a function generates a sampling space:

Proposition 5. ([SZ99], Theorem 1) Let $V(\varphi)$ be a shift invariant space. Then the following two assertions are equivalent:

(i) The space $V(\varphi)$ is a sampling space 
(ii) The function $\varphi$ is continuous, $\sum_{k \in \mathbb{Z}}|\varphi(x-k)|^{2}$ is bounded on $\mathbb{R}$ and

$$
A \chi_{E_{\varphi}}(\omega) \leq\left|Z_{\varphi}(0, \omega)\right| \leq B \chi_{E_{\varphi}}(\omega) \text { a.e. } \omega
$$

for some constants $A, B>0$.

Proposition 6. ([SZ04], Theorem 1.2) Let $V(\varphi)$ be a sampling space with sampling function s. Then there exists a sampling space $V(\tilde{\varphi})$ with sampling function $\tilde{s}$ such that:

1. The space $V(\varphi)$ is a subspace of $V(\tilde{\varphi})$

2. The sequence $\{\tilde{s}(\cdot-k)\}_{k \in \mathbb{Z}}$ is a Riesz basis for $V(\tilde{\varphi})$.

\subsection{Proofs of Theorems 1 and 2}

First we need the following lemmas:

Lemma 1. Assume $V(\varphi) \subset L^{2}(\mathbb{R})$ is a shift invariant space and $\varphi$ is a continuous function. Let $\psi \in V(\varphi)$ such that $\{\psi(\cdot-k)\}_{k \in \mathbb{Z}}$ is a Bessel sequence. If $\sum_{\mathbb{R} .}|\varphi(x+k)|^{2}<L<+\infty \quad \forall x \in \mathbb{R}$, then $\sum_{k \in \mathbb{Z}}|\psi(x+k)|^{2}<L^{\prime}<+\infty \forall x \in$

The proof of this lemma is in [SZ99] for the case that $\{\psi(\cdot-k)\}_{k \in \mathbb{Z}}$ is a frame of $V(\varphi)$, but the same proof works if it is only a Bessel sequence.

Lemma 2. ([Sun05], Lemma 2.5) If $\varphi \in L^{2}(\mathbb{R})$ is continuous and $\sum_{k \in \mathbb{Z}} \mid \varphi(x+$ $k)\left.\right|^{2}<L<+\infty$, then $Z_{\varphi}(x, \omega)=0 \forall x \in \mathbb{R}$, a.e. $\omega \in \mathbb{R} \backslash E_{\varphi}$.

Remark 2. Let $V(\varphi)$ be a sampling space and $s$ its sampling function. For $f \in V(\varphi)$,

$$
\hat{f}(\omega)=Z_{f}(0, \omega) \hat{s}(\omega) \quad \text { a.e. } \omega \in \mathbb{R} .
$$

Therefore we obtain

$$
G_{f}(\omega)=\left|Z_{f}(0, \omega)\right|^{2} G_{s}(\omega) \quad \text { a.e. } \omega \in \mathbb{R} .
$$

To see this, observe that

$$
f(x)=\sum_{k \in \mathbb{Z}} f(k) s(x-k),
$$

with uniform convergence and in $L^{2}(\mathbb{R})$, so

$$
\hat{f}(\omega)=\left(\sum_{k \in \mathbb{Z}} f(k) e^{-2 \pi i k \omega}\right) \hat{s}(\omega)=Z_{f}(0, \omega) \hat{s}(\omega) \quad \text { a.e. } \omega \in \mathbb{R}
$$

and then 


$$
\sum_{k}|\hat{f}(\omega+k)|^{2}=\left|Z_{f}(0, \omega)\right|^{2} \sum_{k}|\hat{s}(\omega+k)|^{2} \quad \text { a.e. } \omega \in \mathbb{R}
$$

hence

$$
G_{f}(\omega)=\left|Z_{f}(0, \omega)\right|^{2} G_{s}(\omega) \quad \text { a.e. } \omega \in \mathbb{R} .
$$

Lemma 3. Let $V(\varphi)$ be a sampling space and $s$ its sampling function. Then we have:

i) If $\psi_{1}, \psi_{2} \in L^{2}(\mathbb{R})$ and $S\left(\psi_{1}\right)=S\left(\psi_{2}\right)$, then $E_{\psi_{1}}=E_{\psi_{2}}$ (up to a set of measure zero). In particular if $\{\psi(\cdot-k)\}_{k \in \mathbb{Z}}$ is a frame for $V(\varphi)$ then $E_{\varphi}=E_{\psi}$.

ii) The sampling function satisfies $Z_{s}(0, \omega)=\chi_{E_{s}}(\omega)$ a.e. $\omega \in \mathbb{R}$.

iii) For $f \in V(\varphi), E_{f}=\left\{\omega \in \mathbb{R}: Z_{f}(0, \omega) \neq 0\right\}$ (up to a set of measure zero).

iv) The sampling function s is unique, up to a set of measure zero, and satisfies $\hat{s}(\omega)=\frac{\psi(\omega)}{Z_{\psi}(0, \omega)} \chi_{E_{\varphi}}(\omega)$ for each generator $\psi$ whose translates form a frame of $V(\varphi)$.

Proof. $i$ ) If $\{\psi(\cdot-k)\}_{k \in \mathbb{Z}}$ is a frame for $V(\varphi)$ then there exists $\left\{c_{k}\right\}_{k \in \mathbb{Z}} \in \ell^{2}(\mathbb{Z})$ such that

$$
\varphi(x)=\sum_{k \in \mathbb{Z}} c_{k} \psi(x-k) .
$$

So $G_{\varphi}(\omega)=\left|\left(\sum_{k \in \mathbb{Z}} c_{k} e^{-2 \pi i k \omega}\right)\right|^{2} G_{\psi}(\omega)$ a.e. $\omega \in \mathbb{R}$, which yields $E_{\varphi} \subseteq E_{\psi}$ (up to a set of measure zero). Similarly we obtain the other inclusion. Now, if $\psi_{1}, \psi_{2}$ generate the same SIS, we can modify these generators as in (2) to obtain tight frames, and the result follows.

ii) Since we have $\hat{s}(\omega+k)=Z_{s}(0, \omega) \hat{s}(\omega+k)$ a.e. $\omega \in \mathbb{R}$, it follows that $Z_{s}(0, \omega)=1$ for almost every $\omega \in E_{s}$. On the other side, by Proposition 5 $Z_{s}(0, \omega)=0$ for almost every $\omega \notin E_{s}$.

iii) We have

$$
G_{f}(\omega)=\left|Z_{f}(0, \omega)\right|^{2} G_{s}(\omega) \quad \text { a.e. } \omega \in \mathbb{R} .
$$

Using Proposition [6 we can assume that $\{s(\cdot-k)\}_{k \in \mathbb{Z}}$ is a Riesz basis of the sampling space, so $G_{s}(\omega) \neq 0$ a.e. $\omega \in \mathbb{R}$, which implies $\left.i i i\right)$.

$i v$ ) Let $\psi$ be a generator whose translates form a frame of $V(\varphi)$. We have $\hat{\psi}(\omega)=Z_{\psi}(0, \omega) \hat{s}(\omega)$ a.e. $\omega \in \mathbb{R}$. By $\left.i\right), E_{\varphi}=E_{\psi}$, so using $\left.i i i\right)$,

$$
\hat{s}(\omega)=\frac{\hat{\psi}(\omega)}{Z_{\psi}(0, \omega)} \text { a.e. } \omega \in E_{\varphi}
$$

and the result follows. 
Notation. Let $f, g \in L^{2}(\mathbb{R})$. We denote

$$
[f, g](x)=\sum_{k \in \mathbb{Z}} f(x+k) \overline{g(x+k)} .
$$

Observe that $G_{f}(\omega)=[\hat{f}, \hat{f}](\omega)$.

Since the Fourier transform preserves the scalar product, if $V$ is a closed subspace of $L^{2}(\mathbb{R})$ and $P_{V}$ is its orthogonal projection, we have that $\widehat{P_{V}(f)}=$ $P_{\hat{V}}(\hat{f})$, where $\hat{V}=\{\hat{f}: f \in V\}$.

In BDR94 the following formula for the orthogonal projection was obtained:

$$
P_{\widehat{S(\varphi)}}(\hat{f})(\omega)=r(\omega) \hat{\varphi}(\omega)
$$

where

$$
r(\omega)= \begin{cases}\frac{[\hat{f}, \hat{\varphi}](\omega)}{[\hat{\varphi}, \hat{\varphi}](\omega)} & \text { for } \omega \in E_{\varphi} \\ 0 & \text { otherwise. }\end{cases}
$$

We are finally ready to prove the theorems.

Proof of Theorem 1. For $f \in V(\varphi)$, we define

$$
\hat{h}(\omega)= \begin{cases}\frac{\hat{f}(\omega)}{Z_{f}(0, \omega)} & \text { for } \omega \in E_{f} \\ 0 & \text { otherwise. }\end{cases}
$$

Observe that by Lemma $3 i v$ ) the function $h$ is well defined. Using Proposition 4 , it is easy to see that $S(f)=S(h)$. To show that $\{h(\cdot-k)\}_{k \in \mathbb{Z}}$ is a frame sequence, first observe that $E_{h}=E_{f}$, so for almost every $\omega \in E_{h}$,

$$
G_{h}(\omega)=\frac{G_{f}(\omega)}{\left|Z_{f}(0, \omega)\right|^{2}}=G_{s}(\omega)
$$

which is uniformly bounded below and above.

Now we will see that $S(f)=V(h)$ is a sampling space:

Since $h \in S(f) \subseteq V(\varphi), h$ is continuous. By Proposition 5 and Lemma 1] $\sum_{k \in \mathbb{Z}}|h(x+k)|^{2}$ is uniformly bounded in $\mathbb{R}$. So by Lemma 2 $Z_{h}(0, \omega)=$ 0 a.e. $\omega \in \mathbb{R} \backslash E_{h}$. Furthermore, for almost every $\omega \in E_{h}$,

$$
G_{h}(\omega)=\left|Z_{h}(0, \omega)\right|^{2} G_{s}(\omega)
$$

Since $E_{h} \subseteq E_{s}$ (up to a set of measure zero), we can write for almost all $\omega \in E_{h}$

$$
\frac{G_{h}(\omega)}{G_{s}(\omega)}=\left|Z_{h}(0, \omega)\right|^{2}
$$


and using that $G_{h}(\omega)$ and $G_{s}(\omega)$ are both bounded above and below in $E_{h}$, we have that $\left|Z_{h}(0, \omega)\right|$ is also bounded above and below. Hence, using Proposition 5. we can conclude that $S(f)$ is a sampling space. Moreover, $h$ is its sampling function. To see this, it suffices to prove that for every $g \in S(f), \hat{g}(\omega)=$ $Z_{g}(0, \omega) \hat{h}(\omega)$. But for $g \in S(f) \subseteq V(\varphi)$,

$$
\hat{g}(\omega)=Z_{g}(0, \omega) \hat{s}(\omega),
$$

and since $\hat{h}(\omega)=\hat{s}(\omega)$ for $\omega \in E_{\varphi}$, we have that $\hat{g}(\omega)=Z_{g}(0, \omega) \hat{h}(\omega)$ for almost every $\omega \in E_{f}$. On the other hand, since $g \in S(f)$, there exists a 1-periodic function $r$, such that $\hat{g}(\omega)=r(\omega) \hat{f}(\omega)$, and therefore the equality also holds for almost every $\omega \in \mathbb{R} \backslash E_{f}$.

Hence

$$
\hat{s_{f}}(\omega)=\hat{h}(\omega)=\hat{s}(\omega) \chi_{E_{f}}(\omega) \text { a.e. } \omega \in \mathbb{R} .
$$

Finally we prove that $\hat{h}$ is actually the projection of $s$ onto $S(f)$, i.e. $\hat{h}=$ $\widehat{P_{S(f)}(s)}$.

For almost every $\omega \in E_{f}$,

$$
\begin{gathered}
\widehat{P_{S(f)}(s)}(\omega)=\frac{[\hat{s}, \hat{f}](\omega)}{[\hat{f}, \hat{f}](\omega)} \hat{f}(\omega)= \\
\frac{\left[\hat{s}, Z_{f}(0, \cdot) \hat{s}\right](\omega)}{[\hat{f}, \hat{f}](\omega)} Z_{f}(0, \omega) \hat{h}(\omega)=\left|Z_{f}(0, \omega)\right|^{2} \frac{[\hat{s}, \hat{s}](\omega)}{[\hat{f}, \hat{f}](\omega)} \hat{h}(\omega)=\hat{h}(\omega),
\end{gathered}
$$

and for almost every $\omega \in \mathbb{R} \backslash E_{f}$, we have

$$
\widehat{P_{S(f)}(s)}(\omega)=\hat{h}(\omega)=0
$$

This completes the proof of Theorem [1.

Theorem 2 is an immediate consequence of Proposition 5

\subsection{Proofs of Theorem 3 and Proposition 1}

Proof of Theorem 13. Let $s$ be the sampling function of $V(\varphi)$.

Assume that $\mathcal{F}=\left\{f_{1}, \ldots, f_{m}\right\} \subset V(\varphi)$ is a determining set for $V(\varphi)$. Recall that by Lemma 3, $E_{\varphi}=E_{s}$ (up to a set of measure zero). Since

$$
G_{f_{i}}(\omega)=\left|Z_{f_{i}}(0, \omega)\right|^{2} G_{s}(\omega) \text { a.e. } \omega \in \mathbb{R},
$$

for almost every $\omega \in E_{f_{i}}, \omega$ belongs to $E_{\varphi}$, so the set $\cup_{i=1}^{m} E_{f_{i}} \backslash E_{\varphi}$ has Lebesgue measure zero. 
On the other side there exist $\hat{\alpha_{1}}, \ldots, \hat{\alpha_{m}}$ 1-periodic measurable functions such that

$$
\hat{\varphi}(\omega)=\sum_{i=1}^{m} \hat{\alpha_{i}}(\omega) \hat{f}_{i}(\omega)
$$

so

$$
G_{\varphi}(\omega)=\sum_{k \in \mathbb{Z}}|\hat{\varphi}(\omega+k)|^{2} \leq \sum_{k \in \mathbb{Z}}\left(\sum_{i=1}^{m}\left|\hat{\alpha}_{i}(\omega) \hat{f}_{i}(\omega+k)\right|\right)^{2} .
$$

Hence the set $E_{\varphi} \backslash \cup_{i=1}^{m} E_{f_{i}}$ has Lebesgue measure zero.

To prove the reciprocal it suffices to show that there exist $\hat{\alpha_{1}}, \ldots, \hat{\alpha_{m}} 1-$ periodic measurable functions such that

$$
\hat{s}(\omega)=\sum_{i=1}^{m} \hat{\alpha}_{i}(\omega) \hat{f}_{i}(\omega) .
$$

Define the sets $B_{i}$ inductively by $B_{1}=E_{f_{1}}$, and for $2 \leq i \leq m, \quad B_{i}=E_{f_{i}} \backslash$ $\bigcup_{j=1}^{i-1} B_{j}$. For $1 \leq i \leq m$ set

$$
\hat{\alpha_{i}}(\omega):= \begin{cases}\frac{1}{Z_{f_{i}}(0, \omega)} & \text { for } \omega \in B_{i} \\ 0 & \text { otherwise. }\end{cases}
$$

Since $\hat{s}(\omega)=\hat{\alpha}_{i}(\omega) \hat{f}_{i}(\omega)$ a.e. $\omega \in B_{i}$, equation (15) holds.

Finally we will see that $V(\varphi)=S\left(f_{1}\right)+\cdots+S\left(f_{m}\right)$. Since

$$
\int_{\mathbb{R}}\left|\hat{\alpha}_{i}(\omega)\right|^{2}\left|\hat{f}_{i}(\omega)\right|^{2} d \omega=\int_{B_{i}}|\hat{s}(\omega)|^{2} d \omega<+\infty
$$

it follows that $\hat{\alpha}_{i} \hat{f}_{i} \in S\left(f_{i}\right)$ (see Propositionष4), so $V(\varphi) \subseteq S\left(f_{1}\right)+\cdots+S\left(f_{m}\right)$.

To show the other inclusion, let $g \in S\left(f_{1}\right)+\cdots+S\left(f_{m}\right)$. Then there exist $\hat{\beta_{1}}, \ldots, \hat{\beta_{m}}$ 1-periodic measurable functions such that $\hat{\beta}_{i} \hat{f}_{i} \in L^{2}(\mathbb{R}), 1 \leq i \leq m$, and $\hat{g}=\hat{\beta_{1}} \hat{f_{1}}+\cdots+\hat{\beta_{m}} \hat{f_{m}}$. So, using that $\hat{f_{i}}(\omega)=Z_{f_{i}}(0, \omega) \hat{s}(\omega)$, we have that $\hat{g}(\omega)=\left(\hat{\beta_{1}}(\omega) Z_{f_{1}}(0, \omega)+\cdots+\hat{\beta_{m}}(\omega) Z_{f_{m}}(0, \omega)\right) \hat{s}(\omega)$. Since $\hat{g} \in L^{2}(\mathbb{R})$ and $\hat{\beta_{1}} Z_{f_{1}}(0, \omega)+\cdots+\hat{\beta_{m}} Z_{f_{m}}(0, \omega)$ is a 1-periodic function, applying again Proposition 4. $g$ belongs to $V(\varphi)$.

Proof of Proposition 1. Since $\varphi$ satisfies that there exist $A, B \geq 0$ such that

$$
A \leq G_{\varphi}(\omega) \leq B \text { a.e. } \omega \in E_{\varphi}
$$

then each $\varphi_{j}$ has the same property in $E_{j}$. We conclude that $\left\{\varphi_{j}(\cdot-k)\right\}_{k \in \mathbb{Z}}$ is a frame sequence. Furthermore, note that $\varphi_{j} \in V(\varphi)$ since $\chi_{E_{j}}$ is 1-periodic and $\chi_{E_{j}} \hat{\varphi} \in L^{2}(\mathbb{R})$. Hence, by by Theorem $1, V\left(\varphi_{j}\right)$ is a sampling space. 
Let us show now that $V(\varphi)=\bigoplus_{j} V\left(\varphi_{j}\right)$. Assume that $f \in V(\varphi)$. Then $\hat{f}=m_{f} \hat{\varphi}$ with $m_{f} \in L^{2}[0,1)$.

So, $\hat{f}=\sum_{j}\left(m_{f} \chi_{E_{j}}\right)\left(\chi_{E_{j}} \hat{\varphi}\right)=\sum_{j} m_{j} \hat{\varphi}_{j}$, where $m_{j}=m_{f} \chi_{E_{j}} \in L^{2}[0,1)$. Then $\hat{f}_{j}:=m_{j} \hat{\varphi}_{j} \in V\left(\varphi_{j}\right)$. That is $f=\sum_{j} f_{j}, f_{j} \in V\left(\varphi_{j}\right)$.

On the other side, assume that $g_{j} \in V\left(\varphi_{j}\right)$ and $\sum_{j} g_{j}=0$. Write $\hat{g}_{j}=\theta_{j} \hat{\varphi}_{j}$ with $\theta_{j} \in L^{2}[0,1)$.

Suppose that for some $r \in \mathbb{N}$, the set $M:=\left\{\omega: \hat{g}_{r}(\omega) \neq 0\right\}$ has positive Lebesgue measure. Since $M \subset E_{r}$ we have that for almost all $\omega \in M$,

$$
0=\sum_{j} \hat{g}_{j}(\omega)=\hat{g}_{r}(\omega)=\theta_{r}(\omega) \hat{\varphi}_{r}(\omega) .
$$

The fact that $\omega \in E_{r}$ implies that for some integer $k, \hat{\varphi}(\omega+k) \neq 0$.

Then we can write $0=\theta_{r}(\omega+k) \hat{\varphi}_{r}(\omega+k)=\theta_{r}(\omega) \hat{\varphi}_{r}(\omega+k)$.

So, $\theta_{r}(\omega)=0$. That is $\theta_{r} \equiv 0$ a.e. in $M$, which is a contradiction. We conclude that $g_{j} \equiv 0$ for all $j$.

Since $\varphi_{j} \in V(\varphi)$, by Theorem 1 $\hat{s_{j}}=\hat{s} \chi_{E_{\varphi_{j}}}=\hat{s} \chi_{E_{j}}$ and this completes the proof.

\subsection{Proof of Theorem 4}

First note that if in Proposition [6 we suppose that the sampling function $s$ of $V(\varphi)$ belongs to $\mathcal{U}$, it follows from the proof that the sampling function $\tilde{s}$ of $V(\tilde{\varphi})$ also belongs to $\mathcal{U}$.

Next we prove the following lemma:

Lemma 4. Let $f$ be a function in $L^{2}(\mathbb{R})$. If $f$ belongs to a sampling space, then the sequence $\{f(k)\}_{k \in \mathbb{Z}}$ belongs to $\ell^{2}(\mathbb{Z})$.

Proof. If $f$ belongs to a sampling space, by Proposition 6 we can assume that $f$ belongs to a sampling space $V(\varphi)$ where the integer translates of the sampling function $s$ is a Riesz sequence. That is, there exist constants $A, B>0$ such that

$$
A \leq \sum_{k}|\hat{s}(\omega+k)|^{2} \leq B \quad \text { a.e. } \omega \in \mathbb{R} .
$$

On the other side, applying Remark 2

$$
G_{f}(\omega)=\left|Z_{f}(0, \omega)\right|^{2} G_{s}(\omega) .
$$

Using (7), the left hand side of the equation

$$
\frac{\sum_{k}|\hat{f}(\omega+k)|^{2}}{\sum_{k}|\hat{s}(\omega+k)|^{2}}=\left|Z_{f}(0, \omega)\right|^{2} \quad \text { a.e. } \omega \in \mathbb{R}
$$


has finite integral in $[0,1]$ and coincides almost everywhere with the right hand side, so the function $Z_{f}(0, w)$ is in $L^{2}[0,1]$ and then the sequence $\{f(k)\}_{k \in \mathbb{Z}}$ belongs to $\ell^{2}(\mathbb{Z})$.

The following result is a version of the Poisson Summation Formula.

Proposition 7. If $f \in \mathcal{U}$ and $\{f(k)\}_{k \in \mathbb{Z}} \in \ell^{2}(\mathbb{Z})$, then:

$$
Z_{f}(0, \omega)=\sum_{k \in \mathbb{Z}} \hat{f}(\omega+k) \quad \text { a.e. } \omega \in[0,1] .
$$

Proof. Observe that the function $\sum_{k \in \mathbb{Z}} \hat{f}(\omega+k) \in L^{1}[0,1]$ and its Fourier coefficients are $\{f(k)\}_{k \in \mathbb{Z}}$ and so this function is in $L^{2}[0,1]$.

Remark 3. If $V(\varphi)$ is a sampling space with sampling function s, then it follows

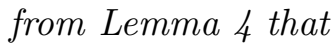

$$
V(\varphi)=\left\{f \in L^{2}(\mathbb{R}):\{f(k)\}_{k \in \mathbb{Z}} \in \ell^{2}(\mathbb{Z}), f(x)=\sum_{k \in \mathbb{Z}} f(k) s(x-k)\right\} .
$$

Proof of Theorem 4. Let $f \in \mathcal{U}$ belong to a sampling space $V(\varphi)$ with $\varphi \in \mathcal{U}$, and let $s$ be its sampling function.

$a)$ is true by Lemma 4 and hence $Z_{f}(0, \omega)$ is well defined, moreover, it is in $L^{2}[0,1]$.

On the other side we have

$$
G_{f}(\omega)=\left|Z_{f}(0, \omega)\right|^{2} G_{s}(\omega) \quad \text { a.e. } \omega \in \mathbb{R},
$$

hence, we can write (by Lemma $3 i v)$ )

$$
\frac{G_{f}(\omega)}{\left|Z_{f}(0, \omega)\right|^{2}}=G_{s}(\omega) \quad \text { a.e. } \omega \in E_{f}
$$

and since $E_{f} \subseteq E_{s}$ (up to a set of measure zero), the right hand side of the preceding equation is bounded above and below by $A$ and $B$ a.e. $\omega \in E_{f}$, and b) follows.

For $c$ ), note that

$$
\int_{E_{f}} \frac{|\hat{f}(\omega)|}{\left|Z_{f}(0, \omega)\right|} d \omega=\int_{E_{f}}|\hat{s}(\omega)| d \omega \leq \int_{-\infty}^{+\infty}|\hat{s}(\omega)| d \omega,
$$

so it suffices to show that $s \in \mathcal{U}$. We have

$$
\hat{\varphi}(\omega)=Z_{\varphi}(0, \omega) \hat{s}(\omega)
$$


By Lemma $3 i), Z_{\varphi}(0, \cdot)$ is bounded below (and above) a.e. in $E_{s}$. Hence

$$
\int_{\mathbb{R}}|\hat{s}(\omega)| d \omega=\int_{\mathbb{R} \cap E_{s}}|\hat{s}(\omega)| d \omega \leq C \int_{\mathbb{R} \cap E_{s}}|\hat{\varphi}(\omega)| d \omega=C \int_{\mathbb{R}}|\hat{\varphi}(\omega)| d \omega,
$$

which proves our claim. We will now prove $d$ ).

By the Fourier Inversion Formula, for all $x \in \mathbb{R}$ and all $k \in \mathbb{Z}$

$$
s(x+k)=\int_{-\infty}^{+\infty} \hat{s}(\omega) e^{2 \pi i \omega(x+k)} d \omega=\int_{0}^{1} \sum_{j \in \mathbb{Z}} \hat{s}(\omega+j) e^{2 \pi i(\omega+j) x} e^{2 \pi i \omega k} d \omega .
$$

Let $h(\omega, x)=\sum_{j \in \mathbb{Z}} \hat{s}(\omega+j) e^{2 \pi i(\omega+j) x}$. Using Proposition 5 and Lemma 1 $\forall x \in \mathbb{R}$,

$$
\sum_{k \in \mathbb{Z}}\left|\int_{0}^{1} h(\omega, x) e^{2 \pi i \omega k} d \omega\right|^{2}=\sum_{k \in \mathbb{Z}}|s(x+k)|^{2}<L<+\infty .
$$

On the other hand,

$$
\int_{0}^{1}|h(\omega, x)| d \omega \leq \int_{-\infty}^{+\infty}|\hat{s}(\omega)| d \omega
$$

so $h(\omega, x) \in L^{1}[0,1]$ as a function of $\omega$.

Then for all $x \in \mathbb{R}, h(\cdot, x) \in L^{2}[0,1]$, and using Parseval's equality, $\|h(\cdot, x)\|_{2}^{2}<$ $L$.

So, since $\hat{s}(\omega)=\frac{\hat{f}(\omega)}{Z_{f}(0, \omega)}$ for $\omega \in E_{f}$, we have

$$
h(\omega, x) \chi_{[0,1] \cap E_{f}}(\omega)=e^{2 \pi i \omega x} \frac{\sum_{s \in \mathbb{Z}} \hat{f}(\omega+s) e^{2 \pi i s x}}{Z_{f}(0, \omega)} \chi_{[0,1] \cap E_{f}}(\omega),
$$

and $d$ ) follows.

This completes the proof of one implication. We will prove now the sufficient conditions. Define

$$
\hat{s}(\omega)= \begin{cases}\frac{\hat{f}(\omega)}{Z_{f}(0, \omega)} & \text { for } \omega \in E_{f} \\ 1 & \text { for } \omega \in[0,1] \backslash E_{f} \\ 0 & \text { otherwise. }\end{cases}
$$

Observe that if $\omega \in E_{f}$, then by condition $\left.b\right)\left|Z_{f}(0, \omega)\right|>0$.

We will prove that $V(s)$ is a sampling space with sampling function $s$ and that $f \in V(s)$. 
First we will see that $\hat{s} \in L^{2}(\mathbb{R})$ and that $\{s(\cdot-k)\}_{k \in \mathbb{Z}}$ is a frame sequence. For almost every $\omega \in E_{f}$,

$$
\sum_{j \in \mathbb{Z}}|\hat{s}(\omega+j)|^{2}=\sum_{j \in \mathbb{Z}} \frac{|\hat{f}(\omega+j)|^{2}}{\left|Z_{f}(0, \omega)\right|^{2}}=\frac{\sum_{j \in \mathbb{Z}}|\hat{f}(\omega+j)|^{2}}{\left|Z_{f}(0, \omega)\right|^{2}}
$$

therefore, using $b$ ), it follows that

$$
A \leq \sum_{k \in \mathbb{Z}}|\hat{s}(\omega+k)|^{2} \leq B \quad \text { a.e. } \omega \in E_{f} .
$$

Now, since by definition of $s, \sum_{k \in \mathbb{Z}}|\hat{s}(x+k)|^{2}=1$ in $\mathbb{R} \backslash E_{f}$, we have that

$$
\min \{1, A\} \leq \sum_{k \in \mathbb{Z}}|\hat{s}(\omega+k)|^{2} \leq \max \{1, B\} \quad \text { a.e. } \omega \in \mathbb{R}
$$

and so $\{s(\cdot-k)\}_{k \in \mathbb{Z}}$ is a frame sequence. (Moreover, this shows that it is a Riesz sequence).

Next we show that $\hat{s} \in L^{1}(\mathbb{R})$ :

$$
\int_{-\infty}^{+\infty}|\hat{s}(\omega)| d \omega=\int_{E_{f}} \frac{|\hat{f}(\omega)|}{\left|Z_{f}(0, \omega)\right|} d \omega+\int_{[0,1] \backslash E_{f}} d \omega .
$$

So by $c), \hat{s} \in L^{1}(\mathbb{R})$ and then $s$ is continuous.

Now we will see that $f \in V(s)$.

$$
\hat{f}(\omega)=Z_{f}(0, \omega) \frac{\hat{f}(\omega)}{Z_{f}(0, \omega)}=Z_{f}(0, \omega) \hat{s}(\omega) \quad \text { a.e. } \omega \in E_{f}
$$

hence using $b$ ) we obtain

$$
\hat{f}(\omega)=Z_{f}(0, \omega) \hat{s}(\omega) \quad \text { a.e. } \omega \in \mathbb{R}
$$

and therefore,

$$
f(x)=\sum_{k \in \mathbb{Z}} f(k) s(x-k) .
$$

To complete the proof, it is enough to verify that the function $s$ satisfies $i i$ ) of Proposition [5. To see that $\sum_{k \in \mathbb{Z}}|s(x+k)|^{2}$ is uniformly bounded, observe 
that for all $x \in \mathbb{R}$ and all $k \in \mathbb{Z}$ we have

$$
\begin{aligned}
s(x+k) & =\int_{-\infty}^{+\infty} \hat{s}(\omega) e^{2 \pi i \omega(x+k)} d \omega \\
& =\int_{E_{f}} \frac{\hat{f}(\omega)}{Z_{f}(0, \omega)} e^{2 \pi i \omega(x+k)} d \omega+\int_{[0,1] \backslash E_{f}} e^{2 \pi i \omega(x+k)} d \omega \\
& =\int_{[0,1] \cap E_{f}} \frac{\sum_{j \in \mathbb{Z}} \hat{f}(\omega+j) e^{2 \pi i(\omega+j)(x+k)}}{Z_{f}(0, \omega)} d \omega+\int_{[0,1] \backslash E_{f}} e^{2 \pi i \omega(x+k)} d \omega .
\end{aligned}
$$

Let us call

$$
g(\omega, x)=\frac{\sum_{j \in \mathbb{Z}} \hat{f}(\omega+j) e^{2 \pi i(\omega+j) x}}{Z_{f}(0, \omega)} \chi_{[0,1] \cap E_{f}}(\omega)+e^{2 \pi i \omega x} \chi_{[0,1] \backslash E_{f}} .
$$

Then

$$
s(x+k)=\int_{0}^{1} g(\omega, x) e^{2 \pi i k \omega} d \omega .
$$

Note that, by $d)$, for all $x \in \mathbb{R}, g(\cdot, x) \in L^{2}[0,1]$ and $\|g(\cdot, x)\|_{2}^{2}<L+1$.

By Parseval's equality we have,

$$
\sum_{k \in \mathbb{Z}}|s(x+k)|^{2}=\|g(\cdot, x)\|_{2}^{2}<L+1 \quad \forall x \in \mathbb{R} .
$$

Since $\{f(k)\}_{k \in \mathbb{Z}} \in \ell^{2}(\mathbb{Z})$ and $\hat{f} \in L^{1}(\mathbb{R})$, the Poisson Summation Formula holds for $f$, that is:

$$
Z_{f}(0, \omega)=\sum_{k \in \mathbb{Z}} \hat{f}(\omega+k) \text { a.e. } \omega \in[0,1] .
$$

Therefore,

$$
\begin{gathered}
s(k)=\int_{-\infty}^{+\infty} \hat{s}(\omega) e^{2 \pi i k \omega} d \omega=\int_{E_{f}} \frac{\hat{f}(\omega)}{Z_{f}(0, \omega)} e^{2 \pi i k \omega} d \omega+\int_{[0,1] \backslash E_{f}} e^{2 \pi i k \omega} d \omega \\
=\int_{[0,1]} e^{2 \pi i k \omega} d \omega=\delta_{0 k},
\end{gathered}
$$

hence $Z_{s}(0, \omega)=1$.

So using Proposition [5 it follows that $V(s)$ is a sampling space. Moreover, by $i v$ ) of Lemma 3, $s$ is its sampling function.

Note that for the proof of the necessary condition of Theorem 4 we did not use that $f \in \mathcal{U}$, hence Proposition 2 is immediate. 


\section{Examples}

Example 1. It is easy to see that the $\sigma$ band-limited functions, for which the Shannon Sampling Theorem holds, satisfy the conditions of Theorem 4.

For instance, let $\sigma=\frac{1}{2}$. Then $\sum_{k \in \mathbb{Z}} \hat{f}(\omega+k)=\hat{f}(\omega) \in L^{2}[0,1]$, so $\{f(k)\}_{k \in \mathbb{Z}} \in$ $\ell^{2}(\mathbb{Z})$. Then, using Poisson Summation Formula, $Z_{f}(0, \omega)=\hat{f}(\omega)$, and conditions b), c) and d) follow.

In SZ04 the following sufficient conditions for a function to belong to sampling space are given:

Proposition 8. (SZ04]) Let $f \in \mathcal{U}$. If there are positive constants $A$ and $B$ such that

$$
A\left|\sum_{k \in \mathbb{Z}} \hat{f}(\omega+k)\right|^{2} \leq \sum_{k \in \mathbb{Z}}|\hat{f}(\omega+k)|^{2}, \quad \text { a.e. }
$$

and

$$
\left(\sum_{k \in \mathbb{Z}}|\hat{f}(\omega+k)|\right)^{2} \leq B\left|\sum_{k \in \mathbb{Z}} \hat{f}(\omega+k)\right|^{2}, \quad \text { a.e. }
$$

then $f$ belongs to a sampling space.

Now we will show that the conditions of our Theorem 4 are weaker than these conditions.

Example 2. Define $f \in L^{2}(\mathbb{R})$ such that:

$$
\hat{f}(\omega)=\sum_{n=0}^{+\infty} \frac{(-1)^{n}}{n+1} \chi_{\left[n, n+\frac{1}{\left.2^{n}\right]}\right.}(\omega) .
$$

Then

$$
\sum_{k \in \mathbb{Z}} \hat{f}(k)=\sum_{n=0}^{+\infty} \frac{(-1)^{n}}{n+1}
$$

and for every $\omega \in\left(1 / 2^{n+1}, 1 / 2^{n}\right]$ :

$$
\begin{gathered}
\sum_{k \in \mathbb{Z}} \hat{f}(\omega+k)=\sum_{k=0}^{n} \frac{(-1)^{k}}{k+1}, \quad \sum_{k \in \mathbb{Z}}|\hat{f}(\omega+k)|=\sum_{k=0}^{n}\left|\frac{1}{k+1}\right| \\
\sum_{k \in \mathbb{Z}}|\hat{f}(\omega+k)|^{2}=\sum_{k=0}^{n}\left|\frac{1}{k+1}\right|^{2} .
\end{gathered}
$$

It is easy to see that $\hat{f} \in L^{1}(\mathbb{R})$ and that $\sum_{k \in \mathbb{Z}} \hat{f}(\omega+k) \in L^{2}[0,1]$. So $\{f(k)\}_{k \in \mathbb{Z}} \in \ell^{2}(\mathbb{Z})$, and using Poisson Summation Formula it follows that $f$ 
satisfies the conditions $b$ ), c) and d) of Theorem 4.

On the other side, $f$ doesn't satisfy the conditions of Proposition 8.

Remark 4. We need to assume that $f, \varphi \in \mathcal{U}$ since in our proof we use the Fourier Inversion Formula and the Poisson Summation Formula.

Note that there exist functions belonging to sampling spaces that are not in $\mathcal{U}$, as we will see in the following example:

Example 3. Let

$$
s(x)= \begin{cases}-\sin (\pi x) & \text { for } x \in[-1,-1 / 2] \\ 1 & \text { for } x \in[-1 / 2,1 / 2] \\ \sin (\pi x) & \text { for } x \in[1 / 2,1] \\ 0 & \text { for } x \notin[-1,1]\end{cases}
$$

This is an example of a sampling function that doesn't belong to $\mathcal{U}$. Applying Lemma 7.3.3 and Corollary 7.3.4 of [Chr03], $\{s(\cdot-k), k \in \mathbb{Z}\}$ is a Riesz basis for the space $V(s)$. Since $s$ is a compactly supported function,

$$
\sum_{k \in \mathbb{Z}}|s(x-k)|^{2}<L<+\infty \quad \forall x \in \mathbb{R}, \text { and } \sum_{k \in \mathbb{Z}} s(k) e^{-2 \pi i k \omega}=s(0)=1 .
$$

Using Proposition [5, $V(s)$ is a sampling space with sampling function s. But $\hat{s} \notin L^{1}(\mathbb{R})$, moreover, for every $f \in V(s), \hat{f} \notin L^{1}(\mathbb{R})$.

\section{ACKNOWLEDGMENT}

The research of the authors is partially supported by Grants PICT03-15033 and UBACYT X058.

The authors want to thank Ursula Molter for reading carefully the manuscript and for her suggestions that helped to improve the paper. Part of this research was carried out while C. Cabrelli and S. Heineken where visiting NuHAG, University of Vienna, and the Erwin Schrödinger International Institute for Mathematical Physics, Vienna, during the special semester on Modern Methods of Time-Frequency Analysis 2005. They want to thank the organizers for their generous hospitality.

\section{References}

[ACHMR03] A. Aldroubi, C. Cabrelli, D. Hardin, U. Molter and A. Rodado, Determining sets of shift invariant spaces, Wavelets and its Applications (Chennai, January 2002), M. Chrisna, R. Radha and S. Thangavelu, eds., Allied Publishers, New Delhi, 1-8, 2003. 
[AG01] A. Aldroubi and K.Gröchenig, Nonuniform sampling and reconstruction in shift-invariant spaces, SIAM Rev. 4, 43 585-620, 2001.

[Bow00] M. Bownik, The structure of shift-invariant subspaces of $L^{2}\left(\mathbb{R}^{n}\right)$, J. Funct. Anal. 2, 177 282-309, 2000.

[BDR94] C. de Boor, R. DeVore and A. Ron, Approximation from shift-invariant subspaces of $L^{2}\left(\mathbb{R}^{d}\right)$, Trans. Amer. Math. Soc. 341, 2 787-806, 1994.

[BF01] J. Benedetto and P. Ferreira, Modern sampling theory, Birkhäuser, Boston, 2004.

[BL98] J. Benedetto and S. Li, The theory of multi-resolution analysis frames and applications to filter banks, Appl. Comp. Harm. Anal., 5 389-427, 1998.

[BZ04] J. Benedetto and A. Zayed, Sampling, wavelets, and tomography, Birkhäuser, Boston, 2001.

[Chr03] O. Christensen, An Introduction to frames and Riesz basis, Birkhäuser, Boston, 2003.

[Gro01] K. Gröchenig, Foundations of Time-Frecuency Analysis, Birkhäuser, Boston, 2001.

[Jan88] A. Janssen, The Zak transform: a signal transform for sampled timecontinuous signals, Philips J. Res. 1, 43, 23-69, 1988.

[Sun05] W. Sun, Sampling theorems for multivariate shift invariant subspaces, Sampl. Theory Signal Image Process. 1, 4, 73-98, 2005.

[SZ99] W. Sun and X. Zhou, On the sampling theorem for wavelet subspaces, J. Fourier Anal. Appl., 5, 347-354, 1999.

[SZ04] W. Sun, and X. Zhou, An Aspect of the Sampling Theorem, International Journal of Wavelets, Multiresolution and Information Processing, 2004. In Press.

[Wal92] G.Walter, A sampling theorem for wavelet subspaces, IEEE Trans. Inform. Theory 2, 38 881-884, 1992. 